\title{
Error-related Brain Activity as a Treatment Moderator and Index of Symptom Change during Cognitive-Behavioral Therapy or Selective Serotonin Reuptake Inhibitors
}

\author{
Stephanie M Gorka*,', Katie L Burkhouse', Heide Klumpp', Amy E Kennedy',2, Kaveh Afshar', \\ Jennifer Francis', Olusola Ajilore', Scott Mariouw', Michelle G Craske ${ }^{3}$, Scott Langenecker', \\ Stewart A Shankman ${ }^{1,4}$ and K Luan Phan ${ }^{1,2,4,5}$ \\ 'Department of Psychiatry, University of Illinois-Chicago, Chicago, IL, USA; ${ }^{2}$ Jesse Brown VA Medical Center, Mental Health Service Line, Chicago, IL, \\ USA; ${ }^{3}$ Department of Psychology, University of California, Los Angeles, Los Angeles, CA, USA; ${ }^{4}$ Department of Psychology, University of Illinois- \\ Chicago, Chicago, IL, USA; ${ }^{5}$ Department of Anatomy and Cell Biology \& the Graduate Program in Neuroscience, University of Illinois-Chicago, \\ Chicago, IL, USA
}

\begin{abstract}
Increased neural error monitoring, as measured by the error-related negativity (ERN), is a transdiagnostic neurobiological marker of anxiety. To date, little is known about whether the ERN can inform the choice between first-line anxiety disorder treatments and whether the ERN changes following treatment completion. The aim of the study was to therefore assess whether the ERN is a treatment moderator and index of symptom change during cognitive-behavioral therapy (CBT) or selective serotonin reuptake inhibitors (SSRIs). Participants included adult volunteers ( $M$ age $=25.8 \pm 8.5 ; 67 \%$ female) with principal anxiety disorders $(n=60)$ or no lifetime history of Axis I psychopathology (ie, healthy controls; $n=26$ ). A flanker task was used to elicit the ERN at baseline and 12 weeks later, following either CBT or SSRIs in the patient sample. Results indicated that baseline ERN was a significant treatment moderator such that a more enhanced baseline ERN was associated with greater reduction in anxiety symptoms within individuals who received CBT but not SSRIs. Results also revealed that the ERN increased pre- to post-treatment among patients randomized to SSRIs, but remained stable among patients randomized to CBT and healthy controls. Together, these novel findings highlight that ERN may help guide treatment decisions regarding engagement in CBT or SSRIs, especially among individuals with an enhanced ERN. The findings also suggest that SSRIs have the capacity to alter individual differences in the ERN, providing evidence that the ERN is not entirely static in patients with anxiety disorders. Neuropsychopharmacology (20I8) 43, I355-1363; doi:10.1038/npp.2017.289; published online I0 January 2018
\end{abstract}

\section{INTRODUCTION}

Increased neural reactivity to errors is a transdiagnostic indicator of anxiety (see Weinberg et al, 2015 for a review). Accordingly, the National Institute of Mental Health (NIMH) Research Domain Criteria (RDoC) Initiative has identified neural error monitoring as a key biologically based dimension of psychological dysfunction that may explain core features of psychopathology, particularly anxiety (Hanna and Gehring, 2016; Weinberg et al, 2015). Neural reactivity to errors is often measured using the error-related negativity (ERN), an event-related potential that appears as a negative-going deflection in the electroencephalogram (EEG) waveform following the commission of an error (Falkenstein et al, 1991; Gehring et al, 1993). The ERN is a well-validated neural response that is observed across various levels of task

\footnotetext{
*Correspondence: Dr SM Gorka, Department of Psychiatry, University of Illinois-Chicago, 1747 W. Roosevelt Road, Chicago, IL 60608, USA, Tel: + I 312413 4707, E-mail: sgorka2@uic.edu

Received 25 July 2017; revised 7 November 2017; accepted 16 November 2017; accepted article preview online 28 November 2017
}

difficulty, response modalities, age ranges, and species (Endrass et al, 2012; Riesel et al, 2013), and therefore reflects activity of a fundamental neural error detection system. In humans, one of the primary sources of the ERN has been localized to the anterior cingulate cortex (Miltner et al, 2003), a neural region known to mediate conflict monitoring and error processing (Botvinick et al, 2004).

To date, an enhanced ERN has been repeatedly observed in individuals with obsessive compulsive disorder (OCD; Ruchsow et al, 2005), generalized anxiety disorder (GAD; Weinberg et al, 2010), and social anxiety disorder (SAD; Endrass et al, 2014). Beyond discrete diagnostic categories, individuals with high trait anxiety (Olvet and Hajcak, 2009), high negative affect (Hajcak et al, 2004), and increased behavioral inhibition (Boksem et al, 2006) are also characterized by an increased ERN. Contributing to this literature, we recently found that greater ERN amplitude was associated with greater levels of fear-based anxiety symptoms (eg, panic, social anxiety), but was unrelated to distress/ misery symptoms (eg, depression), in a heterogeneous comorbid patient population (Gorka et al, 2017) and 
concluded that the ERN may be an objective, psychophysiological marker that tracks the severity of anxiety psychopathology. Of note, however, is that the functional significance of the ERN is still a topic of debate and there are several existing theories that shed light on the mechanisms that may contribute to an enhanced ERN in anxious populations. Researchers have specifically posited that the ERN reflects conflict between two activated response tendencies (Botvinick et al, 2004), a negative reinforcement learning signal generated by phasic activity of the neural dopamine system (Holroyd and Coles, 2002), and a defensive response to the threatening nature of errors (Hajcak and Foti, 2008). Regardless of theoretical differences, most agree that the ERN indexes the functioning of a performance monitoring system designed to flexibly control and modify behavior, and this system tends to be over-active in anxious individuals (Weinberg et al, 2016).

The ERN has been extensively studied with regard to anxiety psychopathology; however, very few studies have addressed the potential role of the ERN in the psychiatry clinic. For instance, a major question within the ERN literature is the degree to which individual differences in ERN predict treatment outcomes and can therefore inform clinical decision-making. Evidence to date is limited to two cognitive-behavioral therapy (CBT) studies with OCD patients, where baseline ERN did not predict symptomatic outcomes (Hajcak et al, 2008) nor differentiate treatment responders from non-responders (Riesel et al, 2015). There have been no studies of treatment prediction with other internalizing disorders or with pharmacological interventions such as selective serotonin reuptake inhibitors (SSRIs). This is noteworthy given that SSRIs are considered a firstline pharmacological treatment for anxiety (Baldwin et al, 2014). Moreover, although both CBT and SSRIs are both effective at reducing internalizing symptoms, treatment response is heterogeneous and the two modalities have some distinct mechanisms that could differentially impact outcomes and treatment prediction (eg, Kennedy et al, 2007). Broadly, CBT is an amalgam of strategies directed at altering maladaptive ways of thinking and coping with negative mood and events (Arch and Craske, 2009), whereas increased serotonin neurotransmission is considered the mechanism of action of SSRIs (Schloss and Williams, 1998). A primary goal of $\mathrm{RDoC}$ is to develop objective dimensional assays that can quantify disease status and guide clinical decision-making, especially between first-line treatment options (Kozak and Cuthbert, 2016). Thus, given the potential for positive predictive findings with respect to disorders other than OCD and with respect to pharmacological treatment, we elected to evaluate ERN as a treatment predictor within anxiety disorder patients in order to advance the mission of precision treatment.

In addition to questions regarding treatment moderation, it is necessary to understand whether the ERN is a static marker of anxiety risk or fluctuates with time and symptom change and could therefore be used as an objective treatment target. As for time, there is some evidence that the ERN is stable over two years in unselected samples (Weinberg and Hajcak, 2011). As for change in symptoms, there have been two studies showing that patients with OCD display an enhanced ERN before and after cognitive CBT (Hajcak et al, 2008; Riesel et al, 2015). Similarly, we found that youth and young adults with SAD had an enhanced ERN relative to healthy controls and individuals with GAD prior to and following treatment with either CBT or SSRIs (Kujawa et al, 2016). Based on existing data, the ERN appears to be a stable anxiety marker that is resistant to internalizing disorder treatments. However, research that extends beyond OCD and SAD diagnostic categories to transdiagnostic symptom change is critically needed to determine whether the ERN has utility as a treatment target.

The current study examined: (1) whether baseline ERN is associated with change in anxiety symptom severity from pre- to post-treatment in a transdiagnostic, anxiety disorder patient population and (2) if the ERN is malleable to change after 12 weeks of either CBT or SSRI treatment. We notably included two separate measures of anxiety symptoms, one capturing broad, non-specific anxiety and the other capturing symptoms specific to fear-based anxiety disorders (ie, panic disorder, SAD, phobias, and post-traumatic stress; Watson, 2005). Based on the existing literature, we hypothesized that the ERN would be relatively stable across time in both healthy controls and individuals with anxiety disorders. We did not have specific hypotheses regarding the association between baseline ERN and change in symptoms with CBT compared to SSRIs given the lack of research in this area.

\section{MATERIALS AND METHODS}

\section{Participants}

The current study was designed to be consistent with, and funded by, the NIMH RDoC Initiative (RFA-MH-13-080). A community sample of individuals with a range of internalizing psychopathologies and symptoms were enrolled if they met criteria for full-threshold or sub-threshold DSM-5 depressive or anxiety disorder and reported a total score of $\geqslant 23$ on the Depression, Anxiety, and Stress Scale (DASS-21; Lovibond and Lovibond, 1995), and a Global Assessment of Functioning score of $\leqslant 60$. Controls had no lifetime Axis I disorders. Exclusionary criteria for all participants included an inability to provide consent and read and write in English; major active medical or neurological problem; lifetime history of mania, psychosis, intellectual disability, or pervasive developmental disorder; current substance dependence; contraindications to receiving SSRIs; engaged in psychiatric treatment including the use of psychiatric medications within the past 4-weeks; traumatic brain injury; and pregnancy. This study was approved by the UIC Institutional Review Board, and informed consent was obtained from all participants.

Given the aims of the current study, only patients with a full-threshold DSM-5 principal anxiety disorder were included in the study (ie, no patient had principal depression). Comorbidities including additional current anxiety disorders and/or major depressive disorder were permitted. A total of 46 healthy controls and 165 patients initially enrolled in the study. For the healthy controls, 10 were deemed ineligible and withdrawn from the study, seven dropped out prior to the baseline assessment, two were lost to follow-up, and one had poor quality ERN data (ie, excessive artifact), resulting in a final sample of 26 controls. For the patients, 47 were deemed ineligible, 36 dropped out 
prior to baseline, 13 were lost to follow-up, and 9 had poor quality ERN data (ie, one made less than six errors (Olvet and Hajcak, 2009); eight had excessive artifact), resulting in a final sample of 60 patients. Of these remaining subjects, 23 received SSRIs and 37 received CBT.

\section{Assessment of Psychopathology}

Lifetime Axis I diagnoses were assessed via the Structured Clinical Interview for DSM-5 Disorders (SCID-5) by a master's degree or $\mathrm{PhD} / \mathrm{MD}$ assessor. A consensus panel of at least three study staff/trained clinicians determined subjects' eligibility and if there were co-occurring disorders, which was the principal disorder warranting treatment. Individuals were not excluded for comorbid disorders but instead classified by their clinician-determined principal diagnosis, as determined by the most severe and impairing symptoms from clinical interviews and self-reports. In the present study, specification of the principal disorder was also used to identify patients with a primary anxiety disorder. Principal anxiety disorders included in the present sample were as follows: $\mathrm{SAD}=20, \mathrm{GAD}=32$, panic disorder $(\mathrm{PD})=3$, and post-traumatic stress disorder $(\mathrm{PTSD})=5$.

At pre- and post-treatment, the Hamilton Anxiety Rating Scale (HAM-A; Hamilton, 1959) was administered by research assessors to measure broad anxiety symptoms (T1 HAMA-A $\alpha=0.89$ ) given that the sample was comprised of individuals with principal anxiety disorders. To capture more specific symptoms, participants also completed the Inventory for Depression and Anxiety Symptoms-II (IDASII; Watson et al, 2012), which includes distinct, factor analytically derived symptom scales that map onto DSM-IV anxiety disorders. Within the current sample, we have previously demonstrated that baseline ERN amplitude is specifically associated with fear-based anxiety symptoms, and not broad anxiety/general distress (Gorka et al, 2017). In our prior paper, fear-based anxiety symptoms were captured using the IDAS-II by Z-scoring and averaging the panic, social anxiety, claustrophobia, traumatic intrusions and avoidance subscales. These scales were chosen, in particular, as they reflect the symptoms of the 'fear disorders' (ie, PD, SAD, phobia, and PTSD), identified in numerous large-scale, factor analytic studies (Slade and Watson, 2006). Given the findings from Gorka et al (2017), and the potentially unique relation between the ERN and fear-based anxiety, we calculated an identical IDAS-II fear dimension in the current study (T1 $\alpha$ for IDAS fear dimension $=0.90$ ).

At the final treatment session, treating clinicians rated participant clinical improvement using the Clinical Global Impressions Scale (CGI; Guy, 1976). Patients with CGI global improvement ratings of 1 or 2 (ie, very much or much improved) were classified as 'treatment responders'.

\section{Treatment Procedures}

Participants were randomized to either 12 weeks of CBT or SSRI treatment. For SSRIs, the dosing schedule was flexible depending on tolerability and aimed to reach target dose by week 8 (eg, 100-200 mg/day for sertraline). The flexibility of the SSRI protocol was designed to match real-world psychiatric practice. Of the 23 patients randomized to SSRIs, 12 were provided sertraline, 8 were provided escitalopram, and 3 were provided paroxetine. SSRI patients attended 2030 min medication management sessions with their study psychiatrist at $0,2,4,8$, and 12 weeks. For CBT, treatment was delivered through 12, once-weekly 60-min sessions led by a PhD-level clinical psychologist using evidence-based manuals for the patient's principal diagnosis and predominant symptoms (eg, Barlow and Craske, 2006; Craske et al, 1992). As per the manualized protocols, sessions began with psychoeducation and CBT orientation and then expanded to include strategies such as cognitive restructuring and behavioral change (eg, exposure exercises, behavioral activation) around the treatment mid-point (ie, week 6). Sessions ended with a focus on relapse prevention.

\section{Flanker Task to Elicit ERN}

At T1 and T2 participants completed a modified version of the original flanker task (Eriksen and Eriksen, 1974) to measure neural activity to error and correct responses (ie, the ERN and correct response negativity (CRN), respectively). For each trial, participants viewed five horizontally aligned arrowheads. For half of the trials, arrows were compatible (' $>>>>>$ ' or ' $<<<<<$ ') and for the other half, the arrows were incompatible (' $>><>>$ ' or ' $<<><<$ '). Participants were instructed to respond as quickly and accurately as possible to indicate the direction of the center arrow (left or right) by pressing the appropriate mouse button. Arrows were presented for $200 \mathrm{~ms}$ and participants were given up to $1800 \mathrm{~ms}$ to respond. Trials were followed by an intertrial interval (1000-2000 ms) during which a fixation cross appeared. The task consisted of 11 blocks of 30 trials (330 total trials). To encourage fast and accurate responding, participants received performance-based feedback at the end of each block. If accuracy was $75 \%$ correct or lower, the message 'Please try to be more accurate' was presented; if accuracy was greater than $90 \%$, the message, 'Please try to respond faster' was displayed; in all other cases, participants saw the message, 'You're doing a great job'.

\section{Electroencephalogram Data Collection and Processing}

Detailed descriptions of our EEG recording and processing procedures have been published elsewhere (eg, Gorka et al, 2017; Gorka et al, 2016). In brief, EEG was recorded during the task using the ActiveTwo BioSemi system (BioSemi, Amsterdam, the Netherlands). Thirty-four standard electrode sites were used and one electrode was placed on each mastoid. Off-line analyses were performed using Brain Vision Analyzer 2 software (Brain Products, Gilching, Germany). Data were re-referenced to the average of the two mastoids and high-pass $(0.1 \mathrm{~Hz})$ and low-pass $(30 \mathrm{~Hz})$ filtered. Data were segmented beginning $500 \mathrm{~ms}$ before each response onset and continuing for $1500 \mathrm{~ms}$. Standard artifact rejection procedures were used (see Gorka et al, 2017; Gorka et al, 2016). Baseline correction for each trial was performed using the 500 to $300 \mathrm{~ms}$ prior to response onset.

ERN data were considered unusable if the EEG data were contaminated by excessive artifact and/or the participant made less than six errors during the flanker task (Olvet and Hajcak, 2009). The ERN and CRN were scored as the average activity on error and correct trials, respectively, from 0 to $100 \mathrm{~ms}$ after response at electrode $\mathrm{Cz}$, consistent with prior 
studies (eg, Meyer et al, 2013) and where ERN amplitude was maximal. The ERN and CRN were highly correlated $(r=0.70, p<0.01)$, as expected. Therefore, to quantify the difference between error and correct trials, we followed guidelines by Meyer et al (2017) and calculated an ERN standardized residual score $\left(\mathrm{ERN}_{\text {resid }}\right)$ by saving the variance leftover in a regression where the CRN was entered predicting the ERN. The $\mathrm{ERN}_{\text {resid }}$ was used as the primary variable in subsequent analyses consistent with our prior pretreatment study (Gorka et al, 2017). (In addition to the $\mathrm{ERN}_{\text {resid, }}$ we conducted all analyses with the formerly standard $\triangle E R N$ difference score (ie, ERN-CRN). The

Table I Demographics and Clinical Characteristics of Healthy Controls and Anxiety Disorder Patients

Healthy controls $(n=26)$

CBT patients $(n=37)$

SSRI patients $(n=23)$

\begin{tabular}{|c|c|c|c|}
\hline \multicolumn{4}{|l|}{ Demographics } \\
\hline Age (years) & $23.5(8.2)_{\mathrm{a}}$ & $27.3(8.8)_{\mathrm{a}}$ & $26.0(8.0)_{\mathrm{a}}$ \\
\hline Sex (\% female) & $53.8 \%$ a & $67.6 \%$ a & $73.9 \%$ a \\
\hline Hispanic ethnicity & $7.7 \%$ & $18.9 \% \mathrm{a}$ & $17.4 \% \mathrm{a}$ \\
\hline \multicolumn{4}{|l|}{ Race } \\
\hline Caucasian & $53.8 \% \mathrm{a}$ & $64.9 \% \mathrm{a}$ & $73.9 \% \mathrm{a}$ \\
\hline African American & $15.4 \% \mathrm{a}$ & $16.2 \% \mathrm{a}$ & $8.7 \%$ \\
\hline Asian & $30.8 \%$ a & $10.8 \%$ b & $8.7 \% \mathrm{~b}$ \\
\hline American Indian or Alaskan Native & $0.0 \%$ & $0.0 \%$ & $4.3 \%$ a \\
\hline Other/Biracial & $0.0 \% a$ & $8.1 \% a$ & $4.3 \% a$ \\
\hline \multicolumn{4}{|l|}{ Comorbid diagnoses } \\
\hline No. of current internalizing disorders & $0.0_{a}$ & $2.7(1.3)_{\mathrm{b}}$ & $2.6(1.1)_{b}$ \\
\hline Principal anxiety disorder & $0.0_{\mathrm{a}}$ & $100.0 \%$ b & $100.0 \%$ \\
\hline Comorbid current anxiety disorder & $0.0 \% a$ & $70.3 \%$ b & $78.3 \%$ b \\
\hline Comorbid current major depressive disorder & $0.0 \%$ & $41.7 \% \mathrm{~b}$ & $43.5 \% \mathrm{~b}$ \\
\hline Past anxiety disorder & $0.0 \%$ a & $40.5 \%$ b & $26.1 \% \mathrm{~b}$ \\
\hline Past major depressive disorder & $0.0 \% a$ & $40.5 \%$ & $21.7 \%$ \\
\hline Current mild substance use disorder & $0.0 \% a$ & $5.4 \% a$ & $4.3 \%$ a \\
\hline Past substance use disorder & $0.0 \%$ a & $13.5 \%$ a & $8.7 \%$ a \\
\hline \multicolumn{4}{|l|}{ Clinical characteristics } \\
\hline HAM-A symptoms at TI & $1.3(1.7)_{\mathrm{a}}$ & I $6.7(6.4)_{\mathrm{b}}$ & $18.6(7.2)_{\mathrm{b}}$ \\
\hline IDAS fear symptoms at TI (Z-score) & $-1.1(0.2)_{a}$ & $0.4(0.9)_{b}$ & $0.6(0.8)_{b}$ \\
\hline HAM-A symptoms at T2 & N/A & $6.0(3.7)_{a}$ & $6.4(6.0)_{a}$ \\
\hline IDAS fear symptoms at T2 (Z-score) & $N / A$ & $-.07(1.1)_{\mathrm{a}}$ & $.11(0.9)_{\mathrm{a}}$ \\
\hline \multicolumn{4}{|l|}{ Flanker task variables } \\
\hline $\mathrm{ERN}_{\text {resid }}$ at $\mathrm{TI}$ & $-0.10(0.9)_{a}$ & $.08(0.8)_{a}$ & $-.16(0.9)_{\mathrm{a}}$ \\
\hline $\mathrm{ERN}_{\text {resid }}$ at $\mathrm{T2}$ & $0.17(0.8)_{a}$ & $-.31(0.9)_{a}$ & $-.03(1.1)_{\mathrm{a}}$ \\
\hline Accuracy at $\mathrm{TI}$ & $90.3 \%(5.2)_{a}$ & $88.4 \%(8.4)_{a}$ & $90.9 \%(8.0)_{a}$ \\
\hline Accuracy at $T 2$ & $89.4 \%(6.1)$ & $91.3 \%(6.6)$ & $92.2 \%(5.5)$ \\
\hline Range of no. errors made at $\mathrm{TI}$ & $6-66$ & $6-|5|$ & $6-143$ \\
\hline Range of no. errors made at $\mathrm{T} 2$ & $7-76$ & $6-78$ & $6-67$ \\
\hline Error congruent trial reaction time at $\mathrm{TI}$ & $303.3(88.3)_{a}$ & $292.3(89.4)_{a}$ & $263.7(86.7)_{a}$ \\
\hline Error congruent trial reaction time at T2 & $219.6(42.5)_{\mathrm{a}}$ & $286.7(53.6)_{a}$ & $364.0(|46 .|)_{b}$ \\
\hline Error incongruent trial reaction time at $\mathrm{TI}$ & $3 \mid 4.7(10 \mid .2)_{a}$ & $315.2(80.9)_{a}$ & $305.0(51.1)_{a}$ \\
\hline Error incongruent trial reaction time at T2 & $290.7(51.1)_{\mathrm{a}}$ & $325.0(95.8)_{\mathrm{a}}$ & $320.3(82.9)_{a}$ \\
\hline Correct congruent trial reaction time at $\mathrm{TI}$ & $321.4(34.1)_{a}$ & $363.6(83.4)_{a}$ & $353.5(44.7)_{a}$ \\
\hline Correct congruent trial reaction time at T2 & $344.8(56.7)_{a}$ & $361.6(73.6)_{a}$ & $358.5(59.5)_{a}$ \\
\hline Correct incongruent trial reaction time at $\mathrm{TI}$ & $375.5(49.6)_{a}$ & $4 \mid 3.1(69.5)_{a, b}$ & $425.8(88.7)_{b}$ \\
\hline Correct incongruent trial reaction time at $\mathrm{T} 2$ & $394.2(66.9)_{a}$ & $411.3(69.1)_{\mathrm{a}}$ & $418.3(58.2)_{a}$ \\
\hline
\end{tabular}

Note: Means (and standard deviations) or percentages with different subscripts (a, b) across rows were significantly different in pairwise comparisons ( $p<0.05$, chisquare test for categorical variables and Tukey's honestly significant difference test for continuous variables). Some participants met criteria for more than one disorder. HAM-A, Hamilton Anxiety Rating Scale; IDAS, Inventory of Depression and Anxiety Symptoms- II. 
pattern of results was identical across the two methods of quantifying the ERN.)

\section{Data Analysis Plan}

We initially conducted a series of planned within-subjects and between-subjects analyses of variance (ANOVAs) to confirm that patients and controls differed at baseline on symptoms and that treatment was successful in reducing selfreported anxiety.

We then assessed whether the $\mathrm{ERN}_{\text {resid }}$ at $\mathrm{T} 1$ moderated change in anxiety symptom severity during CBT and/or SSRIs within patients (only). To do so, we conducted two hierarchical linear regressions with percent change (pre- to post-treatment) of HAM-A or IDAS fear symptoms as the dependent variable. For both models, the $\mathrm{ERN}_{\text {resid }}$ at $\mathrm{T} 1$ and Treatment (2; CBT, SSRI) were entered in Step 1. The $\mathrm{ERN}_{\text {resid }}$ at $\mathrm{T} 1 \times$ Treatment interaction term was entered in Step 2. Significant interactions were followed using standard simple effects approaches.

We next assessed whether the $\mathrm{ERN}_{\text {resid }}$ changed from $\mathrm{T} 1$ to T2 and correlated with anxiety symptom reduction. First, we conducted a Time (2; T1 and T2) $\times$ Treatment (3; no treatment, CBT, SSRI) omnibus ANOVA. Significant interactions were followed using paired-samples $t$-tests within subjects in each treatment arm. Second, to examine correlations with symptom change, we conducted Pearson's correlations between change in the $\mathrm{ERN}_{\text {resid }}$ and change in HAM-A and IDAS fear symptoms from T1 to T2.

\section{RESULTS}

\section{Demographics and Clinical Characteristics}

As expected, patients reported higher levels of HAM-A, $F(1$, $85)=136.23, p<0.01$, and IDAS fear symptoms, $F(1$, $85)=71.15, p<0.01$, relative to controls at pre-treatment. Within patients, HAM-A, $t(59)=12.42, p<0.01$, and IDAS fear symptoms, $t(59)=9.35, p<0.01$, decreased pre- to posttreatment (average reduction in HAM-A: 63.8\% \pm 26.5 ; average reduction in IDAS fear: $26.5 \% \pm 17.8$ ). There were no differences in pre-treatment symptom severity between the CBT and SSRI groups and the extent of symptom reduction on either measure did not differ based on treatment modality, sex, or race ( $p s>0.16$ ). Based on CGI ratings, $70 \%$ of participants responded to treatment.

During the flanker task, participants achieved $89.6 \%$ and $90.9 \%$ accuracy, and committed $34.1 \pm 24.7$ and $29.8 \pm 20.5$ errors at $\mathrm{T} 1$ and $\mathrm{T} 2$, respectively. Group differences in task accuracy and reaction times for congruent and incongruent trials are presented in Table 1. Neither task accuracy nor reaction times correlated with $\mathrm{ERN}_{\text {resid, }} \mathrm{T} 1$ anxiety symptoms, or change in either anxiety symptom measure (all $p s>0.24$ ). Change in $\mathrm{ERN}_{\text {resid }}$ from $\mathrm{T} 1$ to $\mathrm{T} 2$ also did not correlate with change in task accuracy, correct trial reaction time, or incorrect trial reaction time (all ps $>0.16)$.

\section{ERN at T1 as a Treatment Moderator}

Results of the hierarchical linear regressions are displayed in Table 2. For change in IDAS fear symptoms, findings
Table 2 Hierarchical Linear Regression Analyses Examining Whether Baseline ERN $N_{\text {resid }}$ Predicts Change in Anxiety Symptoms Pre- to Post-Treatment

\begin{tabular}{|c|c|c|c|c|c|c|c|c|}
\hline & \multicolumn{4}{|c|}{ Change in HAM-A } & \multicolumn{4}{|c|}{ Change in IDAS Fear } \\
\hline & $\beta$ & $t$ & $p$-value & $R^{2}$ & $\beta$ & $t$ & $p$-value & $R^{2}$ \\
\hline Step 1 & & & & 0.05 & & & & 0.03 \\
\hline TI ERN $N_{\text {resid }}$ & -0.22 & -1.66 & 0.10 & & -0.13 & -0.96 & 0.34 & \\
\hline Arm & -0.11 & -0.81 & 0.42 & & -0.12 & -0.86 & 0.39 & \\
\hline Step 2 & & & & 0.06 & & & & 0.13 \\
\hline TI ERN $_{\text {resid }} \times$ Arm & 0.65 & 0.82 & 0.41 & & $-1.95 *$ & -2.53 & 0.01 & \\
\hline
\end{tabular}

Abbreviations: Arm, medication or cognitive behavioral therapy; TI, baseline
assessment.
* $p<0.05$.

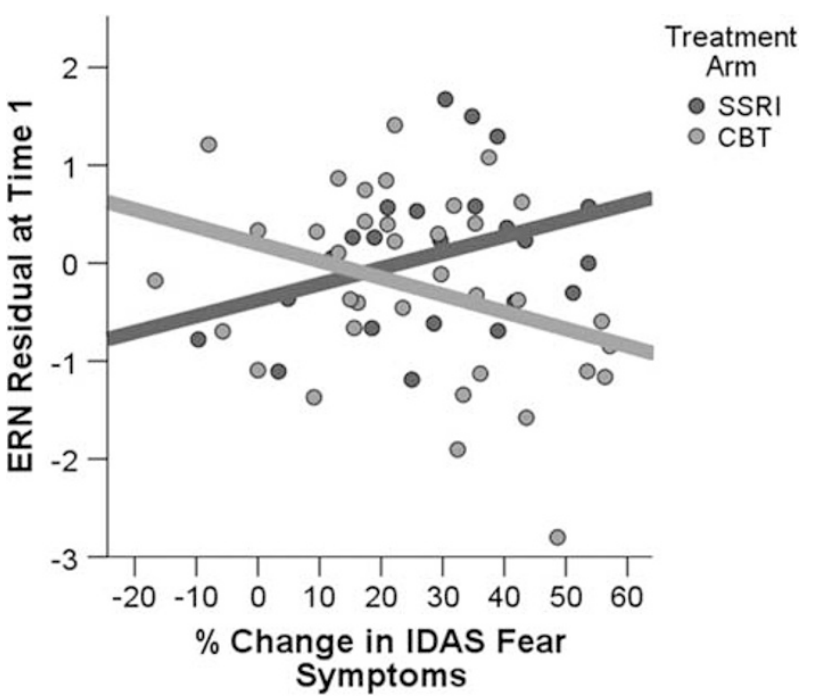

Figure I Scatter plots reflecting the association between pre-treatment ERN and change in IDAS fear symptoms during CBT and SSRIs (separate colors). IDAS, Inventory of Depressive and Anxiety Symptoms; ERN, errorrelated negativity; CBT, cognitive-behavioral therapy; SSRIs, selective serotonin reuptake inhibitors.

revealed no main effects of treatment arm or T1 $\mathrm{ERN}_{\text {resid }}$ but there was a significant treatment arm $\times \mathrm{T} 1 \mathrm{ERN}_{\text {resid }}$ interaction. Follow-up analyses indicated that within patients randomized to CBT, a more enhanced T1 $\mathrm{ERN}_{\text {resid }}$ was associated with greater reduction in fear symptoms $(\beta=$ $-0.34, t=-2.22, p=0.03$; Figure 1). Meanwhile, within patients randomized to SSRIs, there was no association between T1 $\mathrm{ERN}_{\text {resid }}$ and change in fear symptoms $(\beta=0.37$, $t=1.58, p=0.12$ ). (Given the significant symptom overlap and high rates of comorbidity between anxiety and depression (Kessler et al, 2005), we ran an analogous model testing whether baseline ERN amplitude moderated change in depressive symptoms during CBT and/or SSRI treatment using the IDAS depression symptom subscale. Our results did not converge with the anxiety findings as there was no significant baseline $\mathrm{ERN} \times$ treatment arm interaction $(\beta=$ $-.054, t=-0.74, p=0.46)$.) 
Time1 (Week 0)
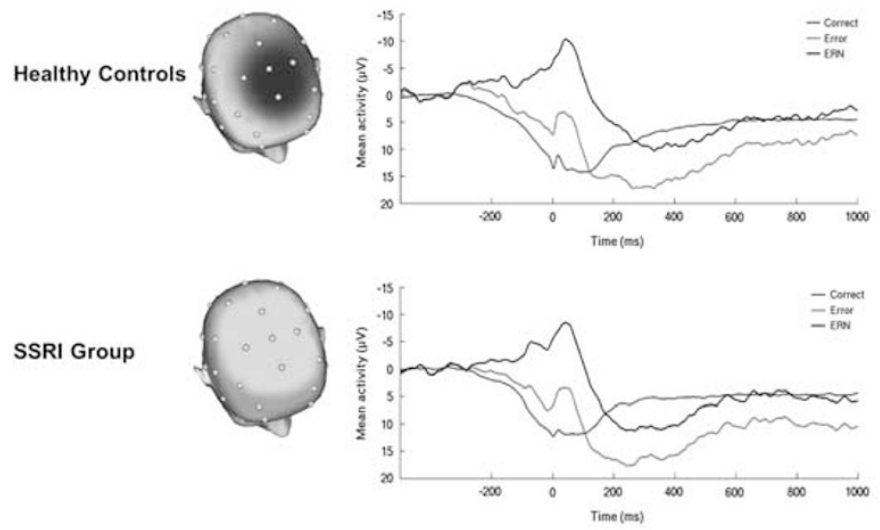

SSRI Group
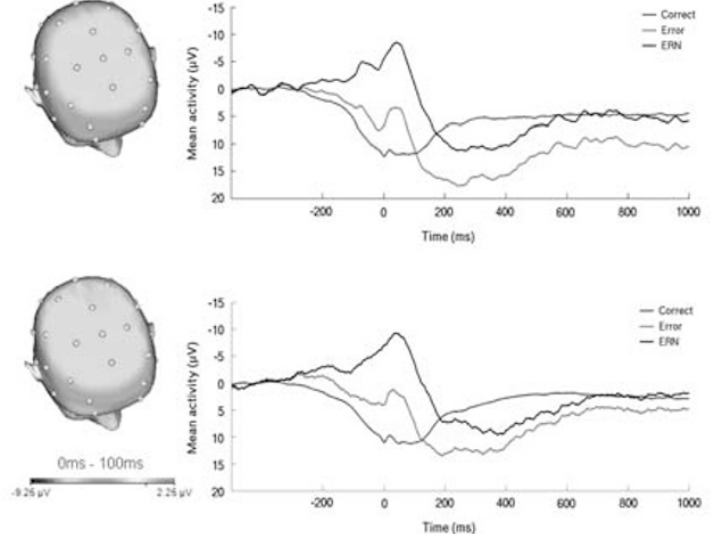
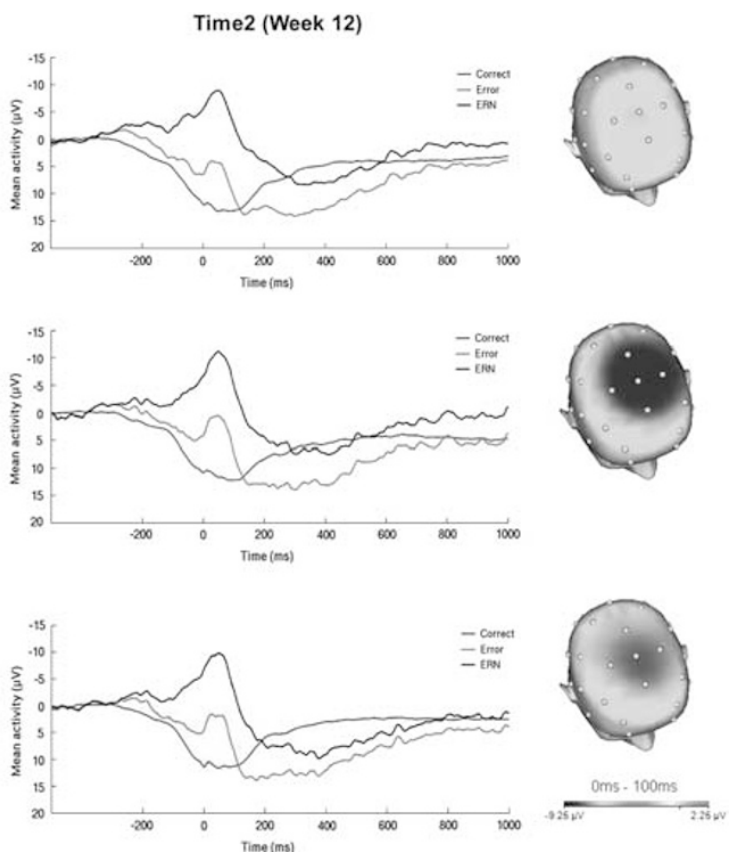

Figure 2 Response-locked ERP waveforms for healthy controls $(n=26)$, individuals randomized to SSRIs $(n=23)$, and individuals randomized to CBT $(n=37)$ at Time I (pre-treatment) and Time 2 (post-treatment). Green lines correspond to correct responses, red lines to errors, and black lines to the $\Delta$ ERN or the error minus correct difference waveform. Topographic scale maps of neural activity depict the error minus correct difference 0-100 ms after the response. CBT, cognitive-behavioral therapy; SSRIs, selective serotonin reuptake inhibitors. A full color version of this figure is available at the Neuropsychopharmacology journal online.

For change in HAM-A symptoms, there were no main effects of treatment arm or T1 $\mathrm{ERN}_{\text {resid. There was also no }}$ significant treatment arm $\times \mathrm{T} 1 \mathrm{ERN}_{\text {resid }}$ interaction.

\section{Change in the ERN Pre- to Post-Treatment}

Results of the omnibus ANOVA indicated that there was no significant main effect of time on the $\mathrm{ERN}_{\text {resid }}(F[1$, $\left.83]=0.03, p=0.86, n p^{2}<0.01\right)$. There was also no main effect of treatment $\left(F[1,83]=0.99, p=0.32, n p^{2}<0.01\right)$; however, there was a time $\times$ treatment interaction $(F[2$, $\left.83]=3.91, p=0.02, n p^{2}=0.09\right)$. Within the healthy controls and patients randomized to $\mathrm{CBT}$, there was no effect of time on the $\mathrm{ERN}_{\text {resid }}$ (controls: $t[25]=-1.73, p=0.10 ; \mathrm{CBT}$ patients: $t[36]=-0.83, p=0.41)$. In contrast, for individuals randomized to SSRIs, $\mathrm{ERN}_{\text {resid }}$ amplitude significantly increased from pre-to-post treatment $(t[23]=1.96, p=0.04)$ (Figure 2).

With regard to symptom change, across all patients, the extent of change in the $\mathrm{ERN}_{\text {resid }}$ from $\mathrm{T} 1$ to $\mathrm{T} 2$ did not correlate with change in HAM-A $(r=-0.03, p=0.85)$ or IDAS fear symptoms $(r=0.09, p=0.48)$. Within the SSRI group specifically, there was also no association between change in $\mathrm{ERN}_{\text {resid }}$ and change in HAM-A $(r=-0.04$, $p=0.88)$ or IDAS fear symptoms $(r=0.28, p=0.24)$.

\section{DISCUSSION}

Results of the current study indicated that within a comorbid anxiety disorder patient population, baseline ERN was a significant treatment moderator such that a more enhanced baseline ERN was associated with greater reduction in fearbased anxiety symptoms within individuals who received
CBT but not SSRIs. Results of the study also indicated that the ERN is modulated by SSRI but not CBT treatment. Among patients randomized to SSRIs, the ERN increased pre- to post-treatment, whereas among patients randomized to CBT and healthy controls, the ERN remained relatively stable. Together, these novel findings highlight that baseline ERN can help guide treatment decisions regarding engagement in CBT or SSRIs. Findings also suggest that SSRIs have the capacity to alter individual differences in the ERN.

The present findings provide important information regarding the utility of the ERN for guiding treatment decision-making. Within individuals randomized to CBT, but not SSRIs, an enhanced ERN was associated with greater reduction in anxiety symptoms, specifically fear-based anxiety and not broad, non-specific anxiety. This treatment moderation finding suggests that individuals with an enhanced pre-treatment ERN who report ongoing fear symptoms such as panic, social anxiety, phobias, and/or traumatic intrusions or avoidance achieve greater levels of symptom relief with CBT than individuals with a more blunted ERN. Patients with an enhanced ERN could therefore be screened and guided towards CBT during treatment decision-making or the ERN could one day be used in a larger objective algorithm for guiding patients to the most appropriate treatment based on their personalized neural and behavioral profile.

The results revealed that an enhanced ERN was associated with preferential CBT outcomes and it is interesting to consider the mechanisms that may underlie this specific finding. Theory and data suggest that cognitive-behavioral therapies achieve fear reduction via inhibitory learning or the development of new inhibitory memories that compete with original excitatory memories to reduce fear expression 
(Craske et al, 2008). Although SSRIs also reduce fear, the mechanisms involve less direct learning and more pharmacological regulation of neural circuitry (Phan et al, 2013). It is possible that individuals with an enhanced ERN achieve better outcomes in the context of structured, new learning and worse outcomes in the absence of such direct manipulations. If conceptualizing the ERN as an indicator of threat sensitivity (Hajcak and Foti, 2008), it is possible that individuals who are highly sensitive to threat display increased CBT engagement and attention towards threat stimuli during CBT exercises which in turn facilitates new learning and reduces fear symptoms (Price et al, 2011). Considering other cognitive theories of the ERN, it is also possible that an increased ERN at baseline reflects an enhanced learning signal and greater working memory and executive capacity (Larson and Clayson, 2011), which sets the stage for increased cognitive gains and improved outcomes within the CBT framework. More specifically, individuals with an enhanced ERN may have better pre-treatment cognitive capabilities and consequently, achieve better CBT outcomes given that $\mathrm{CBT}$ requires memory retention and the ongoing use of cognitive and attentional resources (Mohlman and Gorman, 2005; Wild and Gur, 2008).

We did not originally hypothesize that an enhanced ERN would be associated with change in fear symptoms, only. However, within this sample, we have previously shown that the ERN is more related to current fear-based anxiety than distress/misery symptoms (Gorka et al, 2017), and our broad anxiety measure (ie, HAM-A) notably taps into aspects of depression and distress/misery. It is therefore possible that the ERN is a better predictor of change in fear symptoms because it is a more robust and reliable indicator of the fearbased dimension of psychopathology. Thus, although our findings were observed across individual anxiety disorder diagnoses, pre-treatment ERN screening may be particularly useful for patients presenting to treatment with a fear-based anxiety disorder (eg, PD, SAD) and less useful for distress/ misery anxiety disorders such as GAD. Interestingly, the two prior studies testing whether the ERN is a treatment moderator failed to find significant effects (Hajcak et al, 2008; Riesel et al, 2015) and neither included fear patients or measured fear-based anxiety symptoms suggesting that there may be some specificity to ERN treatment moderation within the anxiety disorder spectrum.

In addition to treatment moderation, the current study also investigated the extent to which individual differences in the ERN change pre- to post-treatment. The ERN is often conceptualized as a stable, trait-like individual difference factor (Olvet and Hajcak, 2008) and indeed, studies have shown that the ERN is stable over 2 years in healthy individuals, is moderately heritable, and related to specific genotypes (Anokhin et al, 2008; Weinberg and Hajcak, 2011). The handful of existing treatment studies have also indicated that the ERN is relatively stable pre- to posttreatment (Hajcak et al, 2008; Riesel et al, 2015) and no differences in ERN amplitude have been observed between medicated (including the use of SSRIs) and unmedicated OCD patients (Stern et al, 2010). However, none of these prior studies have examined the impact of CBT and SSRIs separately and none have included a transdiagnostic anxiety disorder patient sample. The present findings contribute to this literature by corroborating that the ERN is relatively unchanged post-CBT but suggest that SSRIs can increase ERN amplitude in a non-OCD anxiety disorder sample. The ERN is therefore not entirely trait-like and can be manipulated pharmacologically, which is supported by non-treatment studies demonstrating that the ERN is attenuated by alcohol (Ridderinkhof et al, 2002) and enhanced by stimulants (Riba et al, 2005). Notably, the ERN is posited to be mediated by dopamine neurotransmission in the ACC (Holroyd and Coles, 2002), which is why the ERN is sensitive to acute administration of substances such as stimulants. Although SSRIs are not directly linked to the dopaminergic system, dopamine and serotonin are known to interact and SSRIs modulate several dopamine receptor subtypes (Ainsworth et al, 1998; Renard et al, 2001), including D2 receptors in the ACC (Klimke et al, 1999; Larisch et al, 1997). Thus, SSRI's indirect effects on dopamine may have contributed to the present change in ERN pre- to post-SSRIs but not CBT.

We found that SSRIs increased the ERN, not decreased as one may expect. At the same time, within individuals randomized to SSRIs, there was no corresponding increase in anxiety symptoms and treatment outcomes were equivalent in the CBT and SSRI groups. Change in ERN amplitude also did not correlate with change in anxiety symptoms. This pattern of results suggests that there is a disassociation between SSRI-related increases in ERN amplitude and current levels of anxiety symptomatology in patients. It also raises the possibility that the increase in the ERN observed post-SSRIs is a transient phenomenon that reflects dynamic shifts and changes in ACC circuit functioning that are eventually consolidated along with successful symptom reduction. Given that ERN amplitude has been shown to map onto levels of anxiety symptoms (Moser et al, 2013; Gorka et al, in press), it is possible that an exaggerated ERN immediately following SSRI treatment would, over time, decrease to match the level of post-treatment anxiety symptoms and/or anxiety risk. At the same time, if SSRIs do increase ERN amplitude, especially if they do so more chronically, they may serve as a targeted (adjunct) therapy for certain populations that are known to have blunted ERN and poor error processing such as individuals with illicit substance use disorders (Franken et al, 2007) and high levels of externalizing traits (Hall et al, 2007). Given that the present study only included two assessments (pre- and posttreatment), it will be important for future studies to better map the time course of ERN changes following SSRI treatment and assess their clinical utility in samples with known ERN deficits (eg, substance users).

There were several limitations of the current study. First, due to the RDoC strategy of open enrollment of any anxiety disorder there was an unequal number of individual diagnoses which prevents us from examining diagnostic groupings individually. Though this was never the intention of the RDoC approach, future studies may benefit from examining whether these effects are specific for certain diagnostic groups, especially fear-based anxiety disorders. Second, the naturalistic patient sample had high levels of comorbidity. This enhances the external validity of the present findings but also highlights a potential impact of cooccurring psychopathology on the pattern of results. Third, there are several additional factors that are known to influence the ERN (eg, attention-deficit hyperactivity 
disorder (ADHD)) and not all variables were measured and accounted for in the present analyses. Fourth, the present study addressed several aims and therefore included numerous statistical models. Taking this into account, the primary findings would not survive correction for multiple comparisons (eg, Bonferroni correction) and therefore require replication. Lastly, the current study only included two assessment points and in order to accurately model state and trait ERN influences and course of symptoms over time, additional assessment points are needed.

The current study indicates that within anxiety disorder patients, the ERN increases after 12 weeks of SSRIs but not 12 weeks of CBT. The ERN is therefore sensitive to pharmacological manipulation within anxious individuals and it may be possible to target the ERN with novel compounds or drugs to ultimately reduce anxiety symptoms and/or anxiety risk. The findings also indicate that the ERN holds promise as an objective psychophysiological tool for the precision medicine approach as patients with an enhanced, pre-treatment ERN were found to achieve better fear symptom reduction during CBT than individuals with a less enhanced ERN. Given that the ERN is a cost-effective, reliable neural measure, research should continue to explore and validate its use in the clinic.

\section{FUNDING AND DISCLOSURE}

Research reported in this publication was supported by the National Institute of Mental Health of the National Institutes of Health under Award Number R01MH101497 (PI: K. Luan Phan). Other support for this work was provided by the University of Illinois at Chicago (UIC) Center for Clinical and Translational Science (CCTS) award number UL1RR029879 from the National Center for Research Resources. The content is solely the responsibility of the authors and does not necessarily represent the official views of the National Institutes of Health. The remaining authors declare no conflict of interest.

\section{REFERENCES}

Ainsworth K, Smith SE, Zetterstrom TS, Pei Q, Franklin M, Sharp T (1998). Effect of antidepressant drugs on dopamine D1 and D2 receptor expression and dopamine release in the nucleus accumbens of the rat. Psychopharmacology (Berlin) 140: $470-477$.

Anokhin AP, Golosheykin S, Heath AC (2008). Heritability of frontal brain function related to action monitoring. Psychophysiology 45: 524-534.

Arch JJ, Craske MG (2009). First-line treatment: a critical appraisal of cognitive behavioral therapy developments and alternatives. Psychiatr Clin North Am 32: 525-547.

Baldwin DS, Anderson IM, Nutt DJ, Allgulander C, Bandelow B, den Boer JA et al (2014). Evidence-based pharmacological treatment of anxiety disorders, post-traumatic stress disorder and obsessive-compulsive disorder: a revision of the 2005 guidelines from the British Association for Psychopharmacology. J Psychopharmacol 28: 403-439.

Barlow DH, Craske MG (2006). Mastery of your anxiety and panic. Oxford University Press: New York.

Boksem MA, Tops M, Wester AE, Meijman TF, Lorist MM (2006). Error-related ERP components and individual differences in punishment and reward sensitivity. Brain Res 1101: 92-101.
Botvinick MM, Cohen JD, Carter CS (2004). Conflict monitoring and anterior cingulate cortex: an update. Trends Cogn Sci 8: 539-546.

Craske MG, Barlow DH, O'Leary TA (1992). Mastery of your anxiety and worry. Graywind Publications: Albany.

Craske MG, Kircanski K, Zelikowsky M, Mystkowski J, Chowdhury $\mathrm{N}$, Baker A (2008). Optimizing inhibitory learning during exposure therapy. Behav Res Ther 46: 5-27.

Endrass T, Klawohn J, Gruetzmann R, Ischebeck M, Kathmann N (2012). Response- related negativities following correct and incorrect responses: Evidence from a temporospatial principal component analysis. Psychophysiology 49: 733-743.

Endrass T, Riesel A, Kathmann N, Buhlmann U (2014). Performance monitoring in obsessive-compulsive disorder and social anxiety disorder. J Abnorm Psychol 123: 705-714.

Eriksen BA, Eriksen CW (1974). Effects of noise letters upon the identification of a target in a nonsearch task. Percept Psychophys 16: $143-149$.

Falkenstein M, Hohnsbein J, Hoormann J, Blanke L (1991). Effects of crossmodal divided attention on late ERP components. II. Error processing in choice reaction tasks. Electroen Clin Neuro 78: 447-455.

Franken IH, van Strien JW, Franzek EJ, van de Wetering BJ (2007). Error-processing deficits in patients with cocaine dependence. Biol Psychol 75: 45-51.

Gehring WJ, Goss B, Coles MGH, Meyer DE, Donchin E (1993). A neural system for error detection and compensation. Psychol Sci 4: 385-390.

Gorka SM, Burkhouse K, Afshar K, Phan KL (2017). Error-related brain activity and internalizing disorder symptom dimensions in depression and anxiety. Depress Anxiety 34: 985-995.

Gorka SM, MacNamara A, Aase DM, Proescher E, Greenstein JE, Walters $\mathrm{R}$ et al (2016). Impact of alcohol use disorder comorbidity on defensive reactivity to errors in veterans with posttraumatic stress disorder. Psychol Addict Behav 30: 733-742.

Guy W (1972). ECDEU assessment manual for psychopharmacology. US Department of Health, Education, and Welfare, Public Health Service, Alcohol, Drug Abuse, and Mental Health Administration, National Institute of Mental Health, Psychopharmacology Research Branch, Division of Extramural Research Programs 76: 338.

Hajcak G, Foti D (2008). Errors are aversive: defensive motivation and the error-related negativity. Psychol Sci 19: 103-108.

Hajcak G, Franklin ME, Foa EB, Simons RF (2008). Increased errorrelated brain activity in pediatric obsessive-compulsive disorder before and after treatment. Am J Psychiatry 165: 116-123.

Hajcak G, McDonald N, Simons RF (2004). Error-related psychophysiology and negative affect. Brain Cognition 56: 189-197.

Hall JR, Bernat EM, Patrick CJ (2007). Externalizing psychopathology and the error- related negativity. Psychol Sci 18: 326-333.

Hamilton M (1959). The assesment of anxiety states by rating. Brit J Med Psychol 32: 50-55.

Hanna GL, Gehring WJ (2016). The NIMH Research Domain Criteria initiative and error-related brain activity. Psychophysiology 53: 386-388.

Holroyd CB, Coles MG (2002). The neural basis of human error processing: reinforcement learning, dopamine, and the errorrelated negativity. Psychol Rev 109: 679-709.

Kennedy SH, Konarski JZ, Segal ZV, Lau MA, Bieling PJ, McIntyre RS et al (2007). Differences in brain glucose metabolism between responders to CBT and venlafaxine in a 16-week randomized controlled trial. Am J Psychiat 164: 778-788.

Kessler RC, Chiu WT, Demler O, Walters EE (2005). Prevalence, severity, and comorbidity of 12-month DSM-IV disorders in the National Comorbidity Survey Replication. Arch Gen Psychiat 62: 617-627.

Klimke A, Larisch R, Janz A, Vosberg H, Muller-Gartner HW, Gaebel W (1999). Dopamine D2 receptor binding before and after 
treatment of major depression measured by IBZM SPECT. Psychiatry Res 90: 91-101.

Kozak MJ, Cuthbert BN (2016). The NIMH research domain criteria initiative: background, issues, and pragmatics. Psychophysiology 53: 286-297.

Kujawa A, Weinberg A, Bunford N, Fitzgerald KD, Hanna GL, Monk CS et al (2016). Error- related brain activity in youth and young adults before and after treatment for generalized or social anxiety disorder. Prog Neuro-Psychoph 71: 162-168.

Larisch R, Klimke A, Vosberg H, Löffler S, Gaebel W, MüllerGärtner HW (1997). In vivo evidence for the involvement of dopamine-D2 receptors in striatum and anterior cingulate gyrus in major depression. Neuroimage 5: 251-260.

Larson MJ, Clayson PE (2011). The relationship between cognitive performance and electrophysiological indices of performance monitoring. Cogn Affect Behav Neurosci 11: 159-171.

Lovibond SH, Lovibond PF (1995). Manual for the depression anxiety stress scale. 2nd edn, Psychology Foundation: Sydney.

Meyer A, Hajcak G, Torpey DC, Kujawa A, Kim J, Bufferd S et al (2013). Increased error-related brain activity in six-year-old children with clinical anxiety. J Abnorm Child Psychol 41: 1257-1266.

Meyer A, Lerner MD, De Los Reyes A, Laird RD, Hajcak G (2017). Considering ERP difference scores as individual difference measures: Issues with subtraction and alternative approaches. Psychophysiology 54: 114-122.

Miltner WH, Lemke U, Weiss T, Holroyd C, Scheffers MK, Coles MG (2003). Implementation of error-processing in the human anterior cingulate cortex: a source analysis of the magnetic equivalent of the error-related negativity. Biol Psychol 64: 157-166.

Mohlman J, Gorman JM (2005). The role of executive functioning in CBT: a pilot study with anxious older adults. Behav Research Ther 43: 447-465.

Moser JS, Moran TP, Schroder HS, Donnellan MB, Yeung N (2013). On the relationship between anxiety and error monitoring: a meta-analysis and conceptual framework. Front Hum Neurosci 7: 466.

Olvet DM, Hajcak G (2008). The error-related negativity (ERN) and psychopathology: toward an endophenotype. Clin Psychol Rev 28: 1343-1354.

Olvet DM, Hajcak G (2009). The effect of trial-to-trial feedback on the error-related negativity and its relationship with anxiety. Cogn Affect Behav Neurosci 9: 427-433.

Phan KL, Coccaro EF, Angstadt M, Kreger KJ, Mayberg HS, Liberzon I et al (2013). Corticolimbic brain reactivity to social signals of threat before and after sertraline treatment in generalized social phobia. Biol Psychiat 73: 329-336.

Price M, Tone EB, Anderson PL (2011). Vigilant and avoidant attention biases as predictors of response to cognitive behavioral therapy for social phobia. Depress Anxiety 28: 349-353.
Ridderinkhof KR, de Vlugt Y, Bramlage A, Spaan M, Elton M, Snel J et al (2002). Alcohol consumption impairs detection of performance errors in mediofrontal cortex. Science 298: 2209-2211.

Renard CE, Fiocco AJ, Clenet F, Hascoet M, Bourin M (2001). Is dopamine implicated in the antidepressant-like effects of selective serotonin reuptake inhibitors in the mouse forced swimming test? Psychopharmacology 159: 42-50.

Riba J, Rodríguez-Fornells A, Morte A, Münte TF, Barbanoj MJ (2005). Noradrenergic stimulation enhances human action monitoring. J Neurosci 25: 4370-4374.

Riesel A, Endrass T, Auerbach LA, Kathmann N (2015). Overactive performance monitoring as an endophenotype for obsessivecompulsive disorder: evidence from a treatment study. Am J Psychiat 172: $665-673$.

Riesel A, Weinberg A, Endrass T, Meyer A, Hajcak G (2013). The ERN is the ERN is the ERN? Convergent validity of error-related brain activity across different tasks. Biol Psychol 93: 377-385.

Ruchsow M, Grön G, Reuter K, Spitzer M, Hermle L, Kiefer M (2005). Error-related brain activity in patients with obsessive-compulsive disorder and in healthy controls. J Psychophysiol 19: 298-304.

Schloss P, Williams DC (1998). The serotonin transporter: a primary target for antidepressant drugs. J Psychopharmacol 12: $115-121$

Slade TIM, Watson D (2006). The structure of common DSM-IV and ICD-10 mental disorders in the Australian general population. Psychol Med 36: 1593-1600.

Stern ER, Liu Y, Gehring WJ, Lister JJ, Yin G, Zhang J et al (2010). Chronic medication does not affect hyperactive error responses in obsessive-compulsive disorder. Psychophysiology 47: 913-920.

Watson D (2005). Rethinking the mood and anxiety disorders: a quantitative hierarchical model for DSM-V. J Abnorm Psychol 114: $522-536$.

Watson D, O'Hara MW, Naragon-Gainey K, Koffel E, Chmielewski $\mathrm{M}$, Kotov R et al (2012). Development and validation of new anxiety and bipolar symptom scales for an expanded version of the IDAS (the IDAS-II). Assessment 19: 399-420.

Weinberg A, Olvet DM, Hajcak G (2010). Increased error-related brain activity in generalized anxiety disorder. Biol Psychol 85: 472-480.

Weinberg A, Hajcak G (2011). Longer term test-retest reliability of error-related brain activity. Psychophysiology 48: 1420-1425.

Weinberg A, Dieterich R, Riesel A (2015). Error-related brain activity in the age of RDoC: a review of the literature. Int J Psychophysiol 98: 276-299.

Weinberg A, Meyer A, Hale-Rude E, Perlman G, Kotov R, Klein DN et al (2016). Error- related negativity (ERN) and sustained threat: conceptual framework and empirical evaluation in an adolescent sample. Psychophysiology 53: 372-385.

Wild J, Gur RC (2008). Verbal memory and treatment response in post-traumatic stress disorder. Br J Psychiatry 193: 254-255. 